\title{
New Technologies in Intraocular Correction of Aphakia: Assessment of the Long-Term Resulls
}

DOI: $10.17691 /$ stm2018.10.4.16

Received September 13, 2017

A.E. Vasilyeva, Tutor, Department of Eye Diseases;

1.G. Smetankin, MD, DSc, Head of the Department of Eye Diseases

Privolzhsky Research Medical University, 10/1 Minin and Pozharsky Square, Nizhny Novgorod, 603005, Russia

The aim of the study was to assess the long-term results of aphakia correction by implantation of ultra thin intraocular lenses using bimanual phacoaspiration technique.

Materials and Methods. Bimanual phacoaspiration was performed on 16 eyes in 16 patients with intumescent age-related cataracts (maximal follow-up period 5 years). Eleven patients were implanted UltraChoice lenses (ThinOptX, USA), and artificial Incise crystalline lenses (B\&L, USA) were used in the rest five patients.

Results. High stable functional results were noted in the long-term period, vision acuity was 1.0 and higher. All patients had no postoperative complications including induced postoperative astigmatism.

Conclusion. Implantation of ultra thin intraocular lenses in combination with bimanual cataract phacoaspiration may be recommended for a wide clinical application.

Key words: cataract; ultra thin intraocular lens; bimanual phacoaspiration; correction of aphakia.

\section{Introduction}

Lenticular opacity continues to be the most common ophthalmological pathology leading to vision impairment. Operative treatment without intraoperative and especially postoperative complications can completely restore vision functions in patients with cataract [1] Significant advances should be noted in the treatment of this lesion owing to the continuous development of the cataract surgery methods and techniques.

At the current stage, cataract surgeons apply widely small self-sealing tunnel incisions since it is commonly believed that the smaller the incision, the better postoperative results may be achieved by the surgeon in terms of the reduced risk of postoperative complications including induced postoperative astigmatism and a shorter rehabilitation period. Therefore, currently, in spite of the achieved successes, improvements of the lens extraction techniques continue in two main directions: decrease of the operative incision length and the amount of energy used in the course of the operation. Realization of these tasks will minimize the operative trauma and substantially shorten the rehabilitation period [1-4]. One of such methods is bimanual phacoemulcification and phacoaspiration with 0.9-1.4 mm microincisions.

In recent years, the range of intraocular lenses (IOL) produced by foreign and Russian manufactures is constantly expanding. Their material and design is being improved. The development of new more perfect lens models for intraocular correction is carried out with consideration of the tendency in the current ophthalmosurgery: to maximally minimize traumatic injury to the eye tissue. And the most rational way of intraocular aphakia correction is implantation of ultra thin IOLs which are inserted to the eye cavity via 0.9 $1.4 \mathrm{~mm}$ incision. However, for the present, this technique has not been widely used because of the difficulty and complexity of its performance.

The aim of the study was to assess long-term results of using bimanual cataract phakoaspiration and intraocular aphakia correction by ultra thin intraocular lenses in patients with cataract.

\section{Materials and Methods}

Long-term follow-up results are presented for 16 eyes of 16 patients aged from 52 to 65 years $(6$ men and 10 women). Lenticular opacity in the form of intumescent age-related cataract and III and IV degree density of the lens nucleus according to Buratto's classification was diagnosed in all patients.

The study complied with the Declaration of Helsinki (2013) and approved by the Ethical Committee of Privolzhsky Research Medical University. Written informed consent was obtained from every patient.

The maximal follow-up period was 5 years. All patients underwent bimanual phacoaspiration according to the author's technique [5] with implantation of an ultra

Corresponding author: Alina E. Vasilyeva, e-mail: alina-egorova84@mail.ru 
thin IOL. Bimanual phacoaspiration differs from the traditional method (coaxial phacoemulsification) in that the ultrasound energy is not used in the course of the operation, eliminating thereby its negative consequences. Besides, the operation is performed via a microincision $(0.9-1.2 \mathrm{~mm})$, reducing the operative trauma and the rehabilitation period to several days [6].

Eleven patients were implanted artificial UltraChoice lenses (ThinOptX, USA), the recommended length of the surgical incision was up to $1.0 \mathrm{~mm}$, five patients were implanted Incise IOL (B\&L, USA) and the recommended length of the surgical incision was up to $1.4 \mathrm{~mm}$ (Figure 1). All artificial lenses were implanted via $1.2 \mathrm{~mm}$ incision with the help of injectors ThinOptX (Figure 2) and Viscoject $\mathrm{BIO}$ (B\&L, USA). The Infiniti phacoemulsifier (Alcon, USA) equipped with a peristaltic pump and Stellaris phacoemulsifier (B\&L, USA) with a vacuum pump were used in our work. The maximal aspiration level in both units was $600 \mathrm{~mm} \mathrm{Hg}$.

The refractive power was calculated by SRK II formula (M.S. Dang, 1989), KR-8800 refractometer (Topcon, Japan) was used for keratometry, UD8000 ophthalmoscan (Tomey, Lapan) for ultrasound investigations. The IOL refractive power was calculated for commensurable refraction.

The operation was performed in the following way. Two $1.2 \mathrm{~mm}$ surgical incisions were made at 11 and 14 o'clock positions along the upper limbus. After the introduction of viscoelastin, capsulorhexis of the oval form was formed (about $5 \times 6 \mathrm{~mm}$ ) with a capsulotome from the insulin needle, then hydrodissection of the nucleus and epinucleus was performed. Aspiration and irrigation needles were inserted through the incisions. The nucleus was extracted in compliance with the author's technique [6]. The maximal aspiration force realized in the course of the lens nucleus was $400 \mathrm{~mm} \mathrm{Hg}$. Soft lens masses were eliminated using a bimanual aspiration-irrigation system, the anterior and posterior parts of the lens capsule were cleaned with an aspiration cannula. IOL was implanted using an injector via the incision at 11 o'clock position tightly pressing the cannula edges to the external part of the incision (Figure 3). After the completion of the operation, a Floxal eye ointment and Solcoseryl eye gel were placed behind the lower eyelid and an aseptic bandage was applied.

\section{Results and Discussion}

Though the length of the surgical incision was small, Incise IOLs were implanted without any difficulties. In the course of the follow-up, no intra- and postoperative complications were noted in all 5 patients (Figure 4 (a)). In the early and remote period, high vision acuity was achieved (see the Table). The maximal value of induced postoperative astigmatism was $0.25 \mathrm{D}$.
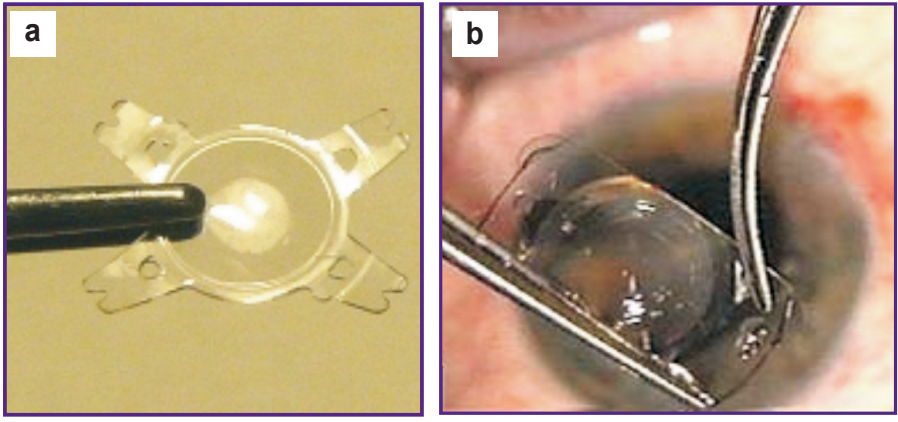

Figure 1. Intraocular lenses:

(a) Incise (B\&L, USA); (b) UltraChoice (ThinOptX, USA)

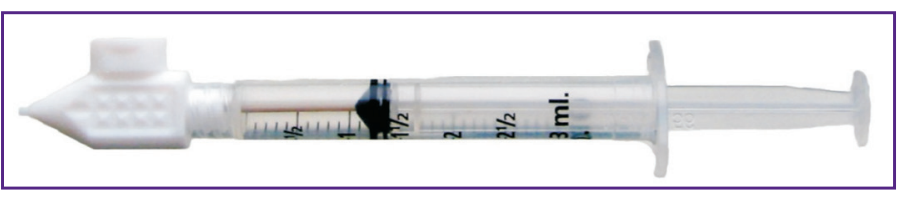

Figure 2. Injector for implantation of ultra thin intraocular lenses

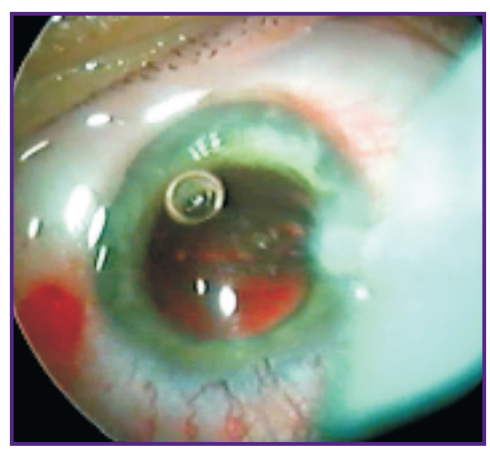

Figure 3. Implantation of the UltraChoice intraocular lens

In 11 patients who were implanted UltraChoice IOLs, in the first $24 \mathrm{~h}$ and later, no injection of conjunctiva and sclera was clinically noted, the cornea was transparent, the moisture of the anterior chamber was without pathologic inclusions, the anterior chamber was deep, the pupils movable (Figure 5 (a)). A slight edema in the zone of surgical incisions not affecting the acuity was noted in 2 patients on day 1 after the operation, single folds of Descemet's membrane were observed (in 2 of them, a slight moisture opalescence of the anterior chamber). Postoperative management was carried out according to the standard schemes, the mentioned events were arrested the next day. All patients were discharged on day 1-2 after the operation, besides individuals of working age resumed their work at once.

Maximal vision acuity at the long-term postoperative period was 0.9 and higher (see the Table). Moreover, it was 1.0 in the majority of patients. Maximal vision acuity in a man of 61 years was 1.25 at the remote period after the implantation of the UltraChoice IOL (Figure 5).

IOL position 5 years after the operation was stable 

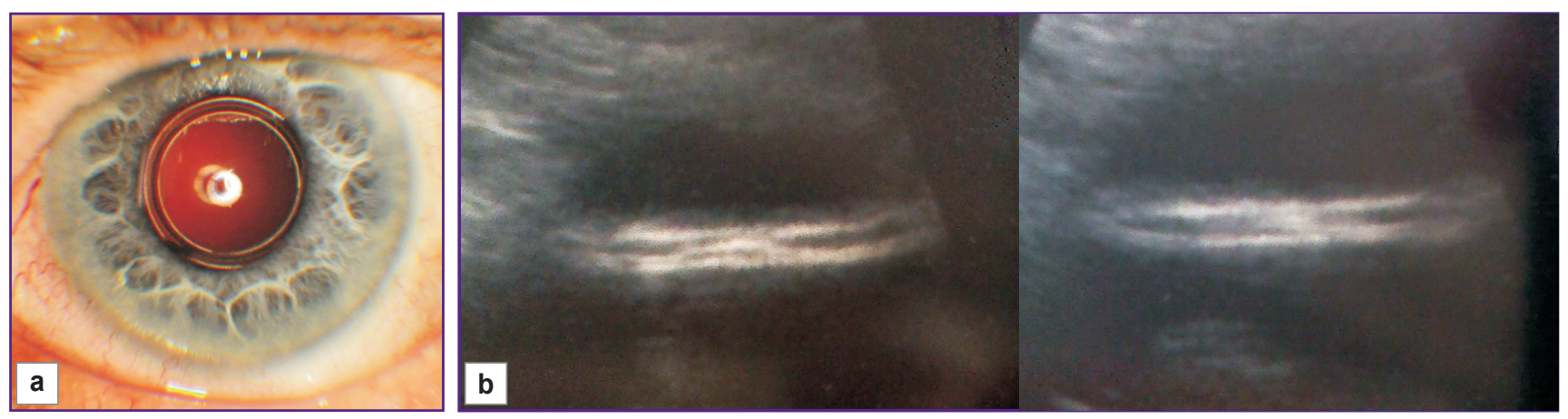

Figure 4. Patient M., 56 years, the right eye:

(a) Incise intraocular lens $24 \mathrm{~h}$ after the operation, vision acuity 1.0; (b) US scanning: left: the direction of probe radiation in the horizontal plane, right: in the vertical plane
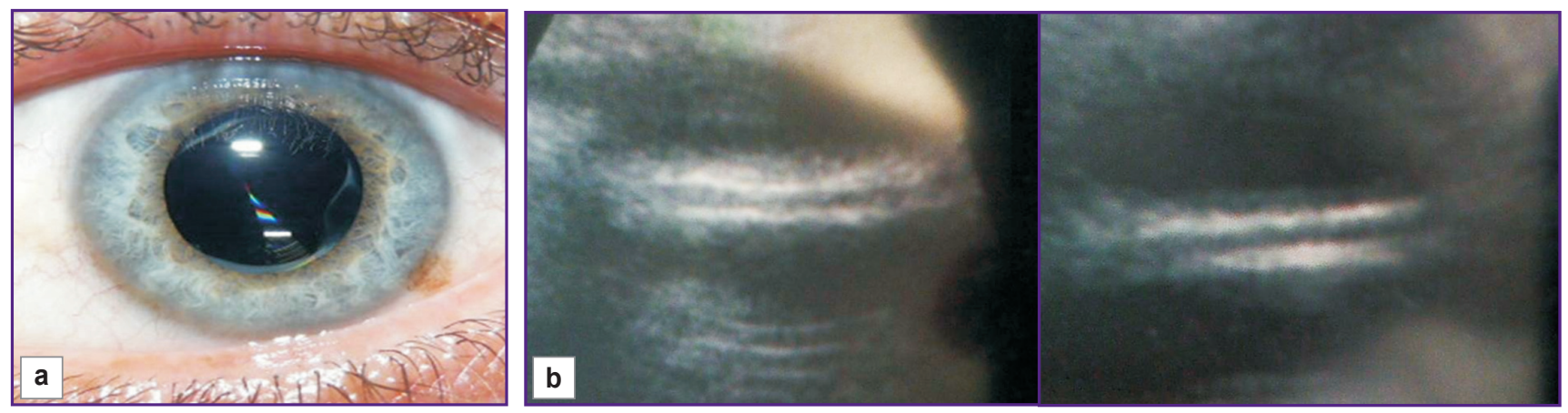

Figure 5. Patient V., 61 years, the left eye:

(a) the UltraChoice intraocular lens, 4.5 years after the operation, vision acuity 1.25; (b) US scanning: left: the direction of probe radiation in the horizontal plane, right: in the vertical plane

Maximal vision acuity in the treated patients at the early and long-term (5 years) period, number of patients (eyes)

\begin{tabular}{lcccccc}
\hline \multirow{1}{*}{$\begin{array}{c}\text { IOL } \\
\text { model }\end{array}$} & \multicolumn{2}{c}{$\begin{array}{c}\text { Vision acuity at the early } \\
\text { postoperative period }\end{array}$} & \multicolumn{3}{c}{$\begin{array}{c}\text { Vision acuity at the long-term } \\
\text { postoperative period }\end{array}$} \\
& $0.8-0.9$ & 1.0 & 1.25 & $0.8-0.9$ & 1.0 & 1.25 \\
UltraChoice & 4 & 7 & - & 3 & 7 & 1 \\
\hline Incise & 2 & 3 & - & 2 & 3 & - \\
\hline
\end{tabular}

in all treated patients, decentrations and dislocations of the artificial lenses were not diagnosed. Gross capsule fibrosis and phimosis were not noted. However, secondary cataract in the form of fibrosis and I degree posterior wall thickening was diagnosed in one patient 3 years after the operation, vision acuity decreased to $0.5 \mathrm{n} / \mathrm{c}$ (no correction). He was performed posterior YAG-laser capsulotomy, acuity vision restored to 1.0 without correction. One case of deviation from the planned refraction (postoperative myopic refraction, sphere 1.0 D) was connected with the errors in the measurements of the eyeball anterior-posterior axis length.

The clinical results obtained were verified by 2D US scanning 5 years later (Figures 4 (b) and 5 (b)). During this examination, no IOL displacements, bends, fractures, and twisting of the optical and haptic part of the artificial lenses were found in all examined patients which speaks of high vision acuity, absence of postoperative induced astigmatism, and the necessity of additional correction to provide maximal acuity.

Thus, at the long-term follow-up period (maximal period 5 years), our patients with intumescent age-related cataracts after bimanual ultrasound phacoemulsification (phacoaspiration) and intraocular correction with ultra thin intraocular lenses were found to have high stable functional results, vision acuity of 0.9 and higher, and absence of complications.

\section{Conclusion}

Application of bimanual phacoemulsification method and subsequent aphakia correction with ultra thin intraocular lenses promotes the reduction of rehabilitation period in patients with cataract and allows the achievement of high stable clinical results at the early and remote postoperative period.

Study funding. The work was not supported by any financial sources. 
Conflicts of interest. The authors have no conflicts of interest to declare.

\section{References}

1. Cataract surgery: maximizing outcomes through research. Bissen-Miyajima H., Koch D.D., Weikert M.P. (editors). Springer Japan 2017, https://doi.org/10.1007/978-4431-54538-5.

2. Mamalis N. Femtosecond laser: the future of cataract surgery? J Cataract Refract Surg 2011; 37(7): 1177-1178, https://doi.org/10.1016/j.jcrs.2011.05.017.

3. Cavallini G.M., Verdina T., De Maria M., Fornasari E., Torlai G., Volante V., Donati S., Cagini C. Bimanual microincision cataract surgery with implantation of the new Incise MJ14 intraocular lens through a $1.4 \mathrm{~mm}$ incision. Int J Ophthalmol 2017; 10(11): 1710-1715, https://doi.org/10.18240/ ijo.2017.11.12.

4. Wang L., Wolfe P., Chernosky A., Paliwal S., Tjia K., Lane S. In vitro delivery performance assessment of a new preloaded intraocular lens delivery system. J Cataract Refract Surg 2016; 42(12): 1814-1820, https://doi.org/10.1016/j. jcrs.2016.10.014.

5. Smetankin I.G. Method of surgical management of cataract. Patent RU 2411023. 2011.

6. Smetankin I.G. New technologies of a lens refractive substitution with a use of microincisions. Sovremennye tehnologii v medicine 2010; 1: 26-30. 